\title{
Cross Domains Arabic Named Entity Recognition System
}

\author{
S. Saad Al-Ahmari*a and B. Abdullatif Al-Johar ${ }^{\mathrm{b}}$ \\ ${ }^{a}$ Computational Bioscience Research Center, King Abdullah University of Science and Technology, \\ Thuwal 23955-6900, Kingdom of Saudi Arabia; ${ }^{\mathrm{b}}$ Computer Science Department, King Faisal \\ University, Hofuf-Al-Hassa-31982,Kingdom of Saudi Arabia
}

\begin{abstract}
Named Entity Recognition (NER) plays an important role in many Natural Language Processing (NLP) applications such as; Information Extraction (IE), Question Answering (QA), Text Clustering, Text Summarization and Word Sense Disambiguation. This paper presents the development and implementation of domain independent system to recognize three types of Arabic named entities. The system works based on a set of domain independent grammar-rules along with Arabic part of speech tagger in addition to gazetteers and lists of trigger words. The experimental results shown, that the system performed as good as other systems with better results in some cases of cross-domains corpora.
\end{abstract}

Keywords: Arabic Natural Language Processing, Named Entity Recognition, Rule Based Approach, Information Extraction, Text Processing.

\section{INTRODUCTION}

The term Named Entity (NE) was introduced in the sixth Message Understanding Conference (MUC-6) [1]. In (MUC-6), the NER consists of three subtypes, Entity Name Expression (ENAMEX) for the proper names, Time Expression (TIMEX) for temporal expressions of time and dates and Numeric Expression (NUMEX) for numeric expressions of percentage, height, monetary expressions, etc. This work focuses on ENAMEX as it's for person, location and organization names.Named Entity Recognition (NER) plays an important role in many Natural Language Processing (NLP) applications such as; Information Extraction (IE), Question Answering (QA), Text Clustering, Text Summarization and Word Sense Disambiguation.NER involves two tasks. The first is the ability to detect the boundary of the named entities from the context. This involves correctly identifying the start and the end tokens for each named entity phrase. The second is the classification of identified names into a set of predefined classes, such as person names, locations and organizations [2].

Rule-based is on of the approaches that are used to recognize NEs such as person names, location names and organization name. It consists of a set of grammar-rules and predefined lists such as gazetteers and trigger words. Based on the comprehensiveness of trigger words and rules rule-based technique can be used to identify NEs in specific domain or in any domain. In this study, we introduce 15 domain independent rules for recognizing person names, location names and organization names in Arabic text. The trigger words are collected from different resources and domains.

A large number of researches have done to tackle NER in different languages. For the Arabic Language only a few of research have focused on it. The complex morphology of Arabic language makes NER a very challenging task. The Arabic language has its unique features and some of the inherent similarities between Arabic and other Semitic languages.

\section{CHALLENGES OF ARABIC NAMED ENTITY RECOGNITION}

The Arabic language considered as one of the highly inflectional languages: Most of single words in Arabic language have more than one affixes such as (Word = prefix (es) + lemma + suffix (es)) [3]. The prefixes include articles, conjunctions or prepositions etc. Lemma refers to the grammatical morpheme of the word. The suffixes include possessive or subjective pronouns, etc. The following example shows a complicated inflection of Arabic word in the word "(وسيكتبونها)" which means "(and they will write it)"; the general form of this world would be " + و

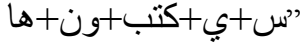


Absence of capital letters in the Arabic orthography: capitalization of the first letter in languages like English, indicating that a word is a proper name. But in the Arabic language, the capital letters are not supported and thus it is hard to detect the NEs [4]. In addition, plenty of person names can also be used as adjective such as: "(Beautiful (جميم (Kind)", "جميلة and "Good) (5].Orthographic with diacritics helps in decrease the ambiguity in Arabic text and the absence of diacritics increases the ambiguity. Diacritics give different meaning to the same word in different context.

There is no standard in writing styles for non-Arabic names translated text. The lack of standardization leads to a proper name may receives different spellings while still corresponds to the same person, organization, or place. A person name like "Jessica" may be translated to Arabic text as: “جاسكا مسكا, جيسيكا جاسيكا, جسيكا" while presenting the same person. Moreover the lack of linguistic resources such as annotated corpora still represents a challenge for Arabic NER [6].

\section{RELATED WORK}

Most of Arabic named entity recognition systems were developed based on rule-based approach. Unfortunately, they used a domain dependent grammar rules. Also, they focus on recognizing person names. There is little attention given to recognize location and organization names. In this section, we present the most related works on Arabic named entity recognition that used rule-based approach.

Abuleil employs a methodology for extracting names from Arabic text using handwritten rules and trigger words, then build a database to store extracted names next to their classification. This procedure passes through three main steps: first: marking the phrases that might involve names, second: collecting information about the words in these phrases and building a graph to represent among them, third: applying rules to recognize and classify the names after that storing names and classes in a database. They collected 500 articles from the Al-Raya newspaper for evaluation. The system accuracy was measured in terms of precision as follow for person $90.4 \%$, for location $93 \%$ and for organization $92.3 \%$ [7].

A parallel corpora (Spanish and Arabic) with Spanish NE tagger used by Samy et al. to recognize Arabic names. A simple mapping scheme has been used to transliterate Arabic text and those words that matched NEs in Spanish text will return as NEs in Arabic. They used a limited size parallel corpus, with 1200 sentence pairs. The output of the NE tagger for each pair compared with the manually annotated gold standard set. They applied a stop word filter to discard the stop words from the possible transliterated candidates. After applying filtering mechanism, their system precision improved from $84 \%$ to $90 \%$ [8].

PERA is a system developed by Shaalan and Raza for person named entity recognition. The main components of their system were a lexicon in form of gazetteer name lists and grammar rules in form of regular. A filtering mechanism was employed within the grammar application, which helps in rejecting matches that appear after person titles but are invalid person names. They have evaluated the performance of their system using semi-automated tagged corpus. The precision was $85.5 \%$ and for $89 \%$ the recall Elsebai collected lists of keywords to identify phrases that may include person names. The main component of their approach is the Arabic Morphological Analyzer (BAMA) used for stemming words. He utilized General Architecture for Text Engineering (GATE) Environment, General Architecture for Text Engineering to apply some natural langue processing tasks such as sentence splitter, tokenization, part-of-speech (POS) tagger and finite state transducer [10]. A set of heuristic rules was introduced to recognize only one type of named entity types: the person name. The formulation of rules depends on the position of words exist in predefined lists and other surrounded words. Introductory Verb List (IVL) and Introductory Word List (IWL) were used. Evaluation corpus consists of 700 articles collected from Al Jazeera website. This system achieved an acceptable f-measure score equal to $89 \%$. [11].

A multilingual NER system was adapted by Zaghouani to handle Arabic using language-independent rules. The recognition of person, location and organization NEs composed of three stages. First, preprocess of Arabic morphology. Second, identify and recognize known NEs. Third, deploy local grammars to recognize unknown NEs. AnerCorp was used for system evaluation. It consists of 316 articles collected from different online news by Benajiba. The overall performance was $73.39 \%$ for precision, $62.13 \%$ for recall and 67.13 for $\mathrm{f}$-measure [12]. 


\section{METHODOLOGY}

In order to make the system domain independent, the researchers divide the process of recognition into several stages and employ different resources. The key stages are: Pre-processing, Part-Of-Speech (POS) tagging, NER annotation and grammar rules. Figure 1shows the system architecture. And how the Arabic text passes through the main components of the system.

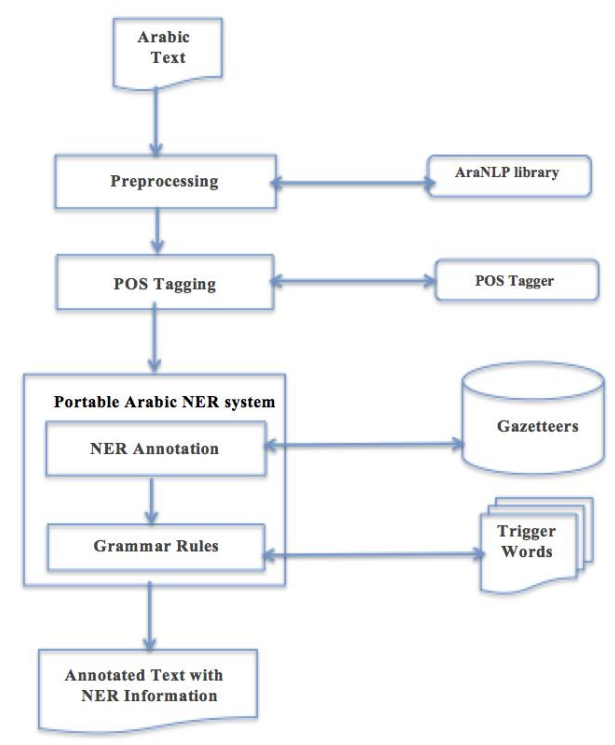

Figure 1. NER System Architecture.

\subsection{Pre-processing}

Normalization takes place after removing punctuations, diacritics and stop words from the text. We used diacritics removal, punctuations removal and Arabic normalizer provided by AraNLP library for text preprocessing. AraNLP developed by Maha Althobiati [13]. Khosrow described stop words as "words having no significant semantic relation to the context in which they exist" [14]. We constructed a list of most frequent stop words that occurred in the selected corpus. It contains prepositions, conjunction, punctuation marks and numbers. Examples of Arabic stop words are: "(In)

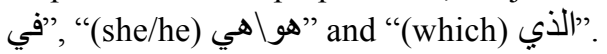

\subsection{POS Tagging}

The task of this stage is to tokenize the normalized text then each token will be tagged based on its features. The tagger used here was developed by Stanford University. Stanford POS tagger is used for tokenization and POS tagging [15]. Words in the text are tagged using the Penn Treebank tag set. Stanford POS tagger exploited maximum entropy methods for the assignment of Penn Treebank tag set. Maximum entropy approach allows the inclusion of diverse sources of information without necessarily assuming independence between the predictors and without causing fragmentation. The best-published accuracy for the tagger on the Penn Treebank is $96.86 \%$ overall and $86.91 \%$ on unknown words. An example of Arabic text tagged by Stanford POS tagger:

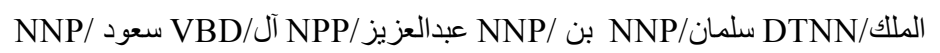

The system relies mainly on the output of this stage since all recognition rules formulated to deal with its output. 


\subsection{NER Annotation}

This stage concerned only with words tagged as proper noun. The aim is to extract and identify named entities based on their POS tags and the gazetteers of Arabic names for person, location and organization. Here a lookup procedure for NEs in the gazetteers is done for NEs annotation. The gazetteers used here developed by Binajiba [16].

ANERgazet were manually built by Benajiba from various web resources:

- Location Gazetteer: it consists of 1,950 names of countries, cities, rivers and mountains found in the Arabic version of Wikipedia.

- Person Gazetteer: it contains of 2,309 person names .The names divided into first names and last names.

- Organizations Gazetteer: it consists of 262 names belong to companies, football teams and other organizations.

All proper nouns, which are not classified by the gazetteers, will be posted to the following stage for processing.

\subsection{Grammar Rules}

This stage is designed to process any proper noun not been resolved by previous stages. A set of domain independent rules is used to extract and classify them into one of the three types of NEs. The set of rules formulated based on analysis of documents from Wikipedia and newspapers articles to identify probable positions of NEs in Arabic text. Each rule describes pattern to match NEs in a given text. Patterns are designed using Regular Expressions [18]. In Arabic sentence person name may preceded by introductory or trigger words such as person titles like "(Dr.) الدكتور "(Mr.) "(السيد ") or verbs such as "(said) قال "اعلن (Announced". Location and organization names could be in different positions in Arabic text and have indicators such as "(university) جامعة "مدينة "or "(city). Lists of trigger words have been collect for the three types of NEs (person, location and organization) from different domains. Trigger words lists are (Person_Trigger_Verbs),(Person_Trigger_Words),(Organization_Preceding_Word),(Organization_Following_Word),(L ocation_Trigger_Words) and (Direction_Trigger_Words). There are 15 domain independent rules for the three types of NEs. Organization rules are examined first then location rules and finally person rules. An organization name may contain person name or location name that is why organization rules applied first. Rules for each type of NEs created from less generalization patterns to more generalization patterns. This mechanism ensures that NEs extracted with correct boundaries. And as a result achieve more comprehensive NEs recognition. Examples of some NEs recognition rules are provided next.

\section{Examples of person name recognition rules:}

$$
\text { ((Person_Trigger_Word) (/[A-Z]))+ ws Person_Name(/NNP//DTNNP)) }
$$

By this rule person name can be recognized if it is preceded by person trigger word like " (doctor)

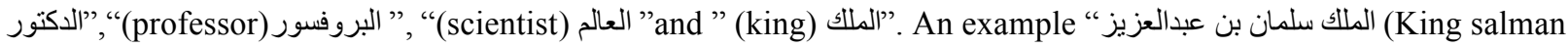
bin Abdulaziz)"

$$
\begin{gathered}
((\text { Person_Trigger_Word }(/[\mathrm{A}-\mathrm{Z}])+\mathrm{ws})+(\text { Person_Ajectives } \mid(\text { conjection }+ \text { Country_Name })(/[\mathrm{A}-\mathrm{Z}])+\mathrm{ws})+ \\
\text { Person_Name }(/ \mathrm{NNP} \mid \mathrm{DTNNP}))
\end{gathered}
$$

By this rule, person name can be recognized, when the person trigger word separated from the person name .The words between them either one or more adjective, or the conjunction and country name. An example for this rule الرئيس الجز ائري عبدالعزيز بوتفليقه (Algerian President Abdualaziz Bouteflika).

\section{Examples of location name recognition rules:}

$$
((\text { Direction_Indicator }(/[\mathrm{A}-\mathrm{Z}]))+\text { ws + Location_Name (/NNP//DTNNP) ) }
$$




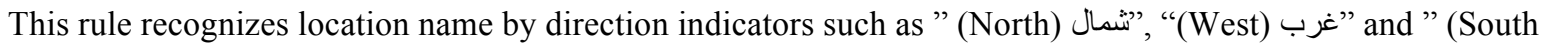
western(Y) يحد المملكة من الجنوب اليمن ". An example (Yemen boarded the Kingdome from south)",

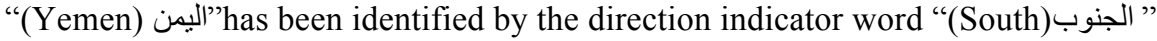

$$
((\text { Location_Trigger_Word (/ [A-Z]) + ws) + Location_Name (/NNP|DTNNP)) }
$$

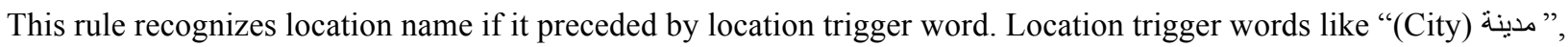

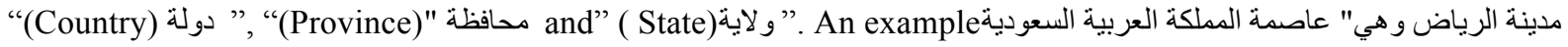

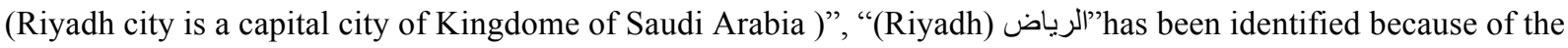
location trigger word " (city)".

\section{Examples of organization name recognition rules:}

(Organization_Preceding_Word $(/[\mathrm{A}-\mathrm{Z}])+\mathrm{ws}+$ Organization_Name $(/ \mathrm{NNP} \mid \mathrm{DTNNP})+($ ws + Organization

$$
\text { Following_Word (/[A-Z]) )?) }
$$

This rule recognizes organization name by looking for words in the organization preceding words and organization

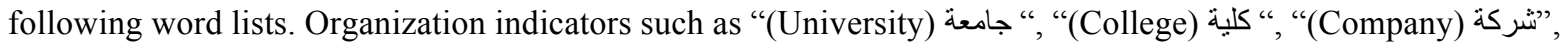

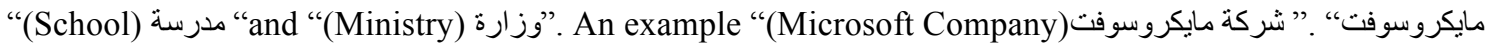

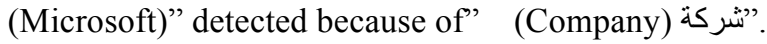

$$
\begin{gathered}
((\text { Organization_Preceding_Word }(/[\mathrm{A}-\mathrm{Z}])+\mathrm{ws})+((\text { Person_Trigger_Word }(/[\mathrm{A}-\mathrm{Z}])))++ \text { Person_Name } \\
(\text { /NNP|DTNNP }))+(\text { ws }+ \text { Organization_Following_Word }(/[\mathrm{A}-\mathrm{Z}])) ?)
\end{gathered}
$$

This rule recognizes organization name, when a person name with its trigger word become part of organization

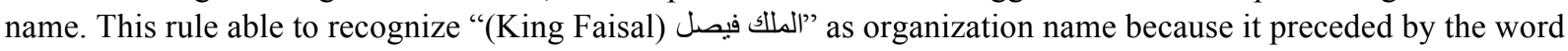
" تأسست جامعة الملك فيصل في عام 1975 ميلادي" in the example) "(University)( جامعة)". 1975)".

\section{SYSTEM EVALUATION}

To prove the system portability cross-domains we test the system performance using corpora from different domains. We evaluate the system using "ANERCorp" which is a corpus developed by Benajiba from different online resources. It consists of 150,000 words along with their tags. ANERcorp is freely available and has become an ideal standard corpus for many Arabic NER research. In order to use ANERcorp corpus for system evaluation, we remove NER annotation from NEs and converted it to integrated raw text. NEs represent $11 \%$ of the whole ANERcorp.

We also evaluate the system using a contemporary corpus developed by Alsuliti. This corpus is a raw text corpus prepared from websites of magazines and newspapers. It consists of deferent categories, more than 8000 words in some categories, identified by the language teachers and language engineers. Corpus categories include politics, health, science and education. In order to use this corpus we converted the articles from XML format to text file format to be more appropriate for research purpose. This corpus is un-annotated corpus therefore we manually annotated the three types of NEs (person, location, organization) for the evaluation process.

The system performance is measured by: precision, recall, and f-measure. These measures are considered standard measures for NER systems [17].To compare the system output for each type of NEs with manually annotated NEs in the corpus we apply "exact- match evaluation". In this scoring protocol, NE is correctly recognized if it exactly matched the corresponding $\mathrm{NE}$ in the corpus. $\mathrm{NE}$ is considered correctly recognized only if the classification is correct and all constituent parts of the NE are detected.

\section{EXPERIMENTS AND RESULTS}

Our objective is to measure the quality of recognition rules and algorithm developed in this work to extract and identify NEs in Arabic text regardless the domain of text. Therefore, we used corpora from different domains to evaluate the performance of the system. The evaluation is done based on two experiments, as we will see in following sections. 


\subsection{Experiment 1}

In this experiment, we examined the system performance against other related works. Hence, we need to use the same corpus used by other's work. Based on our survey we found that the "ANERcorp" corpus developed by Benajiba and Rosso had been used by several systems. Therefore, we chose "ANERcorp" to evaluate the system's results with those performed by others' such as ANERsys 2.0 ( Benajiba and Rosso), RENAR ( Zaghouani, ) and ( Maha Althobaiti ) .

Table 1. The Comparisons between Three Different Systems and Our System when Applied on ANERcorp Corpus

\begin{tabular}{|c|l|l|l|l|l|l|l|l|l|}
\hline \multirow{2}{*}{ Systems } & \multicolumn{3}{|c|}{ Person } & \multicolumn{3}{c|}{ Location } & \multicolumn{3}{c|}{ Organization } \\
\hline & $\mathbf{P}$ & $\mathbf{R}$ & $\mathbf{F}$ & $\mathbf{P}$ & $\mathbf{R}$ & $\mathbf{F}$ & $\mathbf{P}$ & $\mathbf{R}$ & $\mathbf{F}$ \\
\hline $\begin{array}{c}\text { Zaghouani } \\
\text { (RENAR) }\end{array}$ & 71.2 & $\mathbf{5 4 . 2}$ & 61.5 & 90.2 & 85.2 & 87.6 & 58.8 & $\mathbf{4 7}$ & 52.2 \\
\hline $\begin{array}{c}\text { Benajiba } \\
\text { (ANERsys 2.0) }\end{array}$ & 56.3 & 48.6 & 52.1 & 91.7 & 82.2 & 86.7 & 48 & 45.0 & 46.4 \\
\hline $\begin{array}{c}\text { MIthobaiti } \\
\text { (1) }\end{array}$ & $\mathbf{8 5 . 9}$ & 51.2 & $\mathbf{6 4 . 1}$ & 88 & 62.5 & 73.1 & $\mathbf{8 4 . 3}$ & 40.3 & 54.5 \\
\hline \begin{tabular}{c} 
Our System \\
\hline
\end{tabular} & 80.7 & 52.2 & 63.4 & $\mathbf{9 3 . 2}$ & $\mathbf{8 5 . 4}$ & $\mathbf{8 9 . 1}$ & 75.4 & 45.2 & $\mathbf{5 6 . 5}$ \\
\hline
\end{tabular}

From Table 1 we can see that in the location name, our system outperforms the results of the other systems in terms of precision (93.2\%), recall (85.4\%) and f-measure (89.1\%). This is a good indication for a careful lookup procedure. For person and organization names, our system ranked second with precision $80.7 \%$ and $75.4 \%$, respectively. It becomes obvious that all tested systems have difficulties with person and organization types. Person NE type is the most challenging one for Arabic NER systems as some Arabic names have equal weights of probability to be person names, person adjective or place names. For example "Salwa) in sentence like " ذهبت الى سلوى(I went to Salwa) " refers to person name or a city in Saudi Arabia. Therefore, most systems record a low score in precision for person names. ANERsys 2.0 and RENAR systems have achieved only $56.3 \%$ and $71.2 \%$ respectively. The only one peat our system in this type is Althobaiti system as it scores $85.9 \%$. These results may refer to the complexity of the organization names in Arabic as it has many different structures. For instance, a sentence may start with a trigger word for a person name followed by another trigger word for organization then a list of words representing both the person name and the organization name. If we process a sentence like "مدير جامعة الملك فيصل يوسف محمد الجندان (the president of king Faisal university Mohammad Yusif Aljendan)", we will face a problem here as there are two types of names (person and organization) but there is no indication which words representing which type. This kind of sentences is partially recognized if no entry in the gazetteers can resolve this ambiguity.

Although, our system obtained (56.5\%) f-measure for organization NE, it is the highest among the other systems. In general, this experiment proves that the developed algorithm was strong enough to produce better results compared with other systems using the same testing tools. 


\subsection{Experiment 2}

This experiment designed to test the system performance cross-domains. We used a raw text corpus developed by Alsuliti to evaluate the system performance in two phases. In the first phase, we tagged Alsulieti corpus using Stanford POS tagger. As it is clear from Table 2, precision in the recognition of location NE is the highest (94.3\%) among other types of NEs. The reason behind this is because most of the locations are found in the location gazetteer. Also, location names are less ambiguity compared with person or organization names. Organization NE is the second after location NE in term of precision $(90 \%)$.

Table 2. System Performance on Automatic POS tagging.

\begin{tabular}{|c|c|c|c|}
\hline NEs & P & R & F \\
\hline Person & 83.3 & 86.2 & 84.7 \\
\hline Location & 94.3 & 97.3 & 93.7 \\
\hline Organization & 91.5 & 90.7 & 91.1 \\
\hline Overall & 89.7 & 91.4 & 89.8 \\
\hline
\end{tabular}

Second phase, we manually tagged Alsulieti corpus with appropriate POS tags. The purpose of this phase is to be sure that all word assigned a correct POS tag.

Table 3. System Performance on Manually POS tagging.

\begin{tabular}{|c|c|c|c|}
\hline NEs & P & R & F \\
\hline Person & 90.9 & 86.2 & 88.5 \\
\hline Location & 96.2 & 97.3 & 95.6 \\
\hline Organization & 94.7 & 90.7 & 92.7 \\
\hline Overall & 93.9 & 91.4 & 92.3 \\
\hline
\end{tabular}

\section{CONCLUSION AND FUTURE WORK}

We have presented our work on developing a domain independent Arabic NER. The main aim of this work is to use the rule-based approach for NEs recognition includes person, location and organization names in Arabic text. Our method 
consists of three main steps: preprocessing, POS tagging and recognition algorithm. The method had been applied on two Arabic corpora from different domains. The evaluation method has been used to compute the performance metrics for each corpus. The experimental results have shown that the system performed better results in comparison with the other systems using the same testing methods and tools. It also proves the system probabilities cross-domains as it performed good results when it was tested against different domains.

In future work, we intend to expand the used gazetteers and adding more predefined lists to improve system performance. We are also considering the possibility of using different machine-learning methods other than just rulebased technique and how this will improve the overall performance of the system.

\section{REFERENCES}

[1] Grishman, R. and Sumdheim, B. Message Understanding Conference-6: A brief History. in Proceedings of the 16th International Conference on Computational Linguistics, (Copenhagen ,Denmark,1996), 466-471.

[2] Alotaibi, F. and Lee, M. A Hybrid Approach to Features Representation for Fine-grained Arabic Named Entity Recognition. in The 25th International Conference on Computational Linguistic,( Dublin, Ireland,2014),984995.

[3] Habash, N. and Roth, R.M. CATiB: The Columbia Arabic Treebank. in Proceedings of the ACL- IJCNLP 2009 Conference Short Papers,(Suntec, Singapore ,2009), 221- 224.

[4] Mohammed, N.F. and Omar, N. Arabic Named Entity Recognition Using Artificial Neural Network. Journal of Computer Science, 2012, 8(8), 1285-1293.

[5] Koulali, R. and Meziane, A. A Contribution to Arabic Named Entity Recognition. in 10th International Conference on ICT and Knowledge Engineering, (Bangkok, Thailand, 2012), 46-52.

[6] Shaalan, K. A Survey of Arabic Named Entity Recognition and Classification, Association for Computational Linguistics, 2008, 40(2), 470-510.

[7] Abuleil, S. Extracting names from Arabic text for question-answering systems. in Proceedings of Coupling approaches, coupling media and coupling languages for information retrieval, (ParisFrance, 2004), 638-647.

[8] Samy, D., Moreno, A. and Guirao, J. A Proposal for an Arabic Named Entity Tagger Leveraging a Parallel Corpus. in Proceedings of the Conference of the Recent Advances in Natural Language Processing, (Borovets, Bulgaria, 2005), 459-465.

[9] Shaalan, K. and Raza, H. Person Name Entity Recognition for Arabic. in Proceedings of the 5th Workshop on Important Unresolved Matters, (Prague, Czech , 2007), 17-24.

[10] Cunningham, H., Gaizauskas, R. and Wilks, Y. A General Architecture for Text Engineering (GATE) — a new approach to Language Engineering R\&D. Technical Report CS - 95 -21, Department of Computer Science, University of Sheffield, 1995.

[11] Elsebai, A., Meziane, F. Extracting Person Names from Arabic Newspapers. in The International Conference on Innovations in Information Technology, (Abu Dhabi, United Arab Emirates, 2011), 87-89.

[12]Zaghouani, W. Adapting a resource-light highly multilingual Named Entity Recognition system to Arabic. ACM Transactions on Asian Language Information Processing, (New York, USA, 2012), Article 2.

[13] Althobaiti, M., Kruschwitz, U. and Poesio, M. AraNLP: A Java-based Library for the Processing of Arabic Text. in Proceedings of Ninth International Conference on Language Resources and Evaluation, (Reykjavik, Iceland, 2014), 4134-4138.

[14] Alajmi, A., Saad, E.M. and Darwish, R.R. Toward an ARABIC Stop-Words List Generation. International Journal of Computer Applications, 2012, 46(8), 8-13.

[15] Toutanova, K. and Manning, C.D. Enriching the knowledge sources used in a maximum entropy part-of-speech tagger.in Proceedings of the 2000 Joint SIGDAT conference on Empirical methods in natural language processing and very large corpora: held in conjunction with the 38th Annual Meeting of the Association for Computational Linguistics, (Pennsylvania, USA, 2000), 63-70. 
[16] Benajiba, Y. and Rosso, P. ANERsys 2.0: Conquering the NER task for the Arabic language by combining the Maximum Entropy with POS-tag information. in 3rd Indian International Conference on Artificial Intelligence, (Pune, India, 2007), 1814-1823.

[17] Rau, L.F. Extracting Company Names from Text. in Processing of 7th IEEE Conference on Artificial Intelligence Applications,( Florida, USA, 1991), 29-32.

[18] Jagadish, H.V. Li, Y.,Krishnamurthy, R., Raghavan, S. and Vaithyanathan, S. Regular expression learning for information extraction. in Proceedings of the 2008 Conference on Empirical Methods in Natural Language Processing, (Honolulu, USA, 2008), 21-30. 